\title{
A chemokine receptor CCR-1 antagonist reduces renal fibrosis after unilateral ureter ligation
}

\author{
Hans-Joachim Anders, ${ }^{1}$ Volker Vielhauer, ${ }^{1}$ Michael Frink, ${ }^{1}$ Yvonne Linde, ${ }^{1}$ \\ Clemens D. Cohen, ${ }^{1}$ Simone M. Blattner, ${ }^{1}$ Matthias Kretzler, ${ }^{1}$ Frank Strutz, ${ }^{2}$ \\ Matthias Mack, ${ }^{1}$ Hermann-Josef Gröne, ${ }^{3}$ James Onuffer, ${ }^{4}$ Richard Horuk, ${ }^{4}$ \\ Peter J. Nelson, ${ }^{1}$ and Detlef Schlöndorff ${ }^{1}$ \\ ${ }^{1}$ Nephrological Center, Medizinische Poliklinik, Innenstadt, Universität München, München, Germany \\ ${ }^{2}$ Department of Nephrology and Rheumatology, Georg August University Medical Center, Göttingen, Germany \\ ${ }^{3}$ Department of Cellular and Molecular Pathology, German Cancer Research Institute, Heidelberg, Germany \\ ${ }^{4}$ Department of Immunology, Berlex Biosciences, Richmond, California, USA
}

Address correspondence to: Hans-Joachim Anders, Medizinische Poliklinik

Universität München 8a, 80336 München, Germany.

Phone: 49-89-5996856; Fax: 49-89-5996860; E-mail: hjanders@clinbio.med.unimuenchen.de.

Hans-Joachim Anders and Volker Vielhauer contributed equally to this work.

Received for publication August 22, 2001, and accepted in revised form November 26, 2001.

\begin{abstract}
The expression of chemokines and their receptors is thought to contribute to leukocyte infiltration and progressive renal fibrosis after unilateral ureter obstruction (UUO). We hypothesized that blocking the chemokine receptor $\mathrm{CCR} 1$ using the nonpeptide antagonist $\mathrm{BX} 471$ could reduce leukocyte infiltration and renal fibrosis after UUO. UUO kidneys from BX471-treated mice (day $0-10$ and day 6-10) revealed a $40-60 \%$ reduction of interstitial macrophage and lymphocyte infiltrate compared with controls. Treated mice also showed a marked reduction of CCR1 and CCR5 mRNA levels, and FACS analysis showed a comparable reduction of $\mathrm{CD8}^{+} / \mathrm{CCR}^{+} \mathrm{T}$ cells. Markers of renal fibrosis, such as interstitial fibroblasts, interstitial volume, mRNA and protein expression for collagen I, were all significantly reduced by BX471-treatment compared with vehicle controls. By contrast treatment was ineffective when the drug was supplied only from days 0 to 5 . In summary, blockade of CCR1 substantially reduces cell accumulation and renal fibrosis after UUO. Most interestingly, late onset of treatment is also effective. We therefore conclude that CCR1 blockade may represent a new therapeutic strategy for reducing cellular infiltration and renal fibrosis as major factors in the progression to end-stage renal failure.
\end{abstract}

J. Clin. Invest. 109:251-259 (2002). DOI:10.1172/JCI200214040.

\section{Introduction}

Progressive fibrosis leading to loss of organ function is an unsolved issue in clinical medicine. In the kidney, tubulointerstitial fibrosis is the main predictor for the progression to end-stage renal disease (1). Renal fibrosis is characterized by a mixed tubulointerstitial leukocytic cell infiltrate, fibroblast proliferation, and increased matrix production leading to tubular cell necrosis and apoptosis (2). During this process, infiltrating macrophages and lymphocytes are a major source of inflammatory mediators such as cytokines, nitric oxide, and growth factors. Inhibition of leukocyte infiltration may reduce production of such mediators and may therefore be an option to halt progressive renal fibrosis and to prevent or to delay end-stage renal disease.

The leukocytic cell infiltrate is triggered by locally secreted chemokines (3). There is increasing evidence from human renal biopsy studies that the expression of certain chemokine receptors on the surface of leukocytes infiltrating the tubulointerstitium plays an important role in the progression of renal disease (4-6). Unilateral ureteral obstruction (UUO) is a widely used model for progressive renal fibrosis that is independent of hypertension or systemic immune disease (7). Recently, we have characterized the expression of chemokines and their respective receptors during progressive renal fibrosis after UUO in the mouse (8). We found that increasing amounts of the CC-chemokine receptor-1 (CCR1), as well as its chemokine ligands RANTES/CCL5, MIP-1 $\alpha /$ CCL3, and MIP-1ß/CCL4, were expressed in parallel with the progression of renal fibrosis. Since CCR1 is known to mediate the migration of lymphocytes and macrophages into inflamed tissue, CCR1 appeared to be a promising target for a blocking strategy (3). Recently, a potent series of CCR1 antagonists was developed that is also effective on rodent CCR1. The lead compound BX471 - 5-chloro2-(2-[(2R)-4-(4-fluorobenzyl)-2-methylpiperazin-1-yl]-2oxoethoxy phenyl) urea hydrogen chloride salt - is currently in phase I human trials and has been shown to effectively ameliorate disease in a rat experimental allergic encephalomyelitis model and to delay the rejection of rat heart and rabbit renal transplants (9-11). We therefore hypothesized that blocking CCR1 with BX 471 could reduce leukocyte infiltration and renal fibrosis after UUO, a hypothesis supported by our results. 


\section{Methods}

Cell lines and CCR1-expressing cells. The cell lines 293MR (human CCR1/293 cells) and 293M3X (mouse CCR1/293 cells) have been described previously $(12,13)$. The 293M3X/pEAK10-Gqi5 cell line was created by transfecting 293M3X cells with the plasmid pEAK10Gqi5 using Lipofectamine (Life Technologies Inc., Carlsbad, California, USA) and selecting with $0.5 \mu \mathrm{g} / \mathrm{ml}$ puromycin (Edge BioSystems Inc., Gaithersburg, Maryland, USA). pEAK10-Gqi5 directs the overexpression of the chimeric $\mathrm{G}$ protein Gqi5, allowing enhanced cytosolic calcium flux responses from Gi-coupled receptors (12, 13). pEAK10-Gqi5 was constructed by ligating a HinDIII/NotI fragment from plasmid pLEC-Gqi5-HA (Molecular Devices Corp., Sunnyvale, California, USA) into HinDII/NotI-digested pEAK10 (Edge BioSystems Inc.).

Chemokine-binding studies. HEK 293 cells stably expressing human (293MR) or murine (293M3X) CCR1 were grown to confluent monolayers in T-225 square-centimeter flasks as described previously (14). Cells were tested for their ability to bind ${ }^{125}$-labeled MIP-1 $\alpha /$ CCL3 and biological responses by changes in intracellular $\mathrm{Ca}^{2+}$. The binding assays were performed in transfected cells by oil centrifugation methods as described previously (15). Nonspecific binding was determined in the presence of either $100 \mathrm{nM}$ or $1 \mu \mathrm{M}$ unlabeled ligand. The binding data were curve fitted with the computer program IGOR (Wavemetrics, Lake Oswego, Oregon, USA) to determine the affinity and number of sites.

Cytosolic $\mathrm{Ca}^{2+}$ measurements. Cytosolic $\mathrm{Ca}^{+}$measurements in HEK 293 cells overexpressing human and murine (293M3X pEAK10-Gqi5) CCR1 were carried out as follows. Cells were lifted from flasks using an enzymefree cell dissociation buffer (PBS based; Life Technologies Inc.). The cells were then loaded with fluo-3 by resuspension at 1 to $2 \times 10^{6}$ cells $/ \mathrm{ml}$ in HBSS, $20 \mathrm{mM}$ HEPES (all Life Technologies Inc.), $3.2 \mathrm{mM} \mathrm{CaCl}_{2}, 1 \%$ heat-inactivated FBS, $2.5 \mathrm{mM}$ probenecid, $0.04 \%$ Pluronic F-127, $4 \mu \mathrm{M}$ fluo-3, $\mathrm{pH} 7.4$, and incubated at $37^{\circ} \mathrm{C}$ for $1-1.5$ hours. The cells were pelleted by centrifugation and resuspended at $10^{6}$ cells $/ \mathrm{ml}$ in HBSS, 20 mM HEPES, 1 $\mathrm{mM} \mathrm{CaCl} 2,1 \%$ heat-inactivated FBS, $2.5 \mathrm{mM}$ probenecid, $\mathrm{pH} 7.4$, and kept at $37^{\circ} \mathrm{C}$ for analysis. Chemokine-induced cytosolic calcium transients were collected using a thermostated Deltascan 4000 fluorometer running FeliX version 1.41 (Photon Technologies International Inc., Severna Park, Maryland, USA). Animal studies. C57BL/ 6 mice $12 \pm 2$ weeks of age were obtained from Charles River Deutschland $\mathrm{GmbH}$, Sulzfeld, Germany. All mice were kept in macrolone type III cages under a 12-hour light and dark cycle. Water and standard chow (Sniff, GmbH, Soest, Germany) were available ad libitum.

Determination of pharmacokinetic parameters. Male mice were subcutaneously dosed with BX471 $(20 \mathrm{mg} / \mathrm{kg})$ in a vehicle. The vehicle solution was prepared as follows: $40 \%$ cyclodextrin in (\#33,260-7; Sigma-Aldrich Chemie $\mathrm{GmbH}$, Deisenhofen, Germany) unbuffered saline was mixed, dissolved overnight, and filtered through a $0.45-\mu \mathrm{m}$ filter. $\mathrm{BX} 471$ was added at a concentration of $20 \mathrm{mg} / \mathrm{ml}, \mathrm{pH} 3.3$. After complete dissolution, the $\mathrm{pH}$ was raised using $1 \mathrm{~N} \mathrm{KOH}$ to $\mathrm{pH} 4.5$ and filtered as above. Blood samples were withdrawn at various times, and plasma was stored frozen until analyzed. Plasma samples were analyzed either by HPLC using ultraviolet detection methods as described previously (13).

Unilateral ureter ligation. UUO was performed as described previously (8). Mice were sacrificed 10 days after UUO. Eight mice were sacrificed at day 6 . Contralateral kidneys served as intraindividual control. All experimental procedures were performed according to the German animal care and ethics legislation and were approved by the local government authorities.

In group I, control mice were treated subcutaneously with $50 \mu \mathrm{l}$ of the vehicle for 10 days at 8-hour intervals. The first dose was administered immediately after UUO. Group II mice were treated with $50 \mathrm{mg} / \mathrm{kg}$ BX471 in 50 $\mu \mathrm{l}$ vehicle as above from day 1 to 5 at 8 -hour intervals (9). In group III C57BL/ 6 mice received BX471 in vehicle from day 6 to 10, and in group IV C57BL/ 6 mice were treated with BX471 in vehicle from day 1 to 10 .

Morphological evaluation. From each mouse the obstructed and contralateral kidneys were fixed in $4 \%$ formalin in PBS and embedded in paraffin. Two-micrometer sections were stained with periodic acid-Schiff reagent and silver following the instructions of the supplier (Bio-Optica, s.p.a., Milano, Italy). To count interstitial cells 12 highpower fields (hpf's; $\times 400$ ) were analyzed by a blinded observer. Positive cells were counted per hpf omitting positive cells in glomerular fields. Quantitation of the interstitial volume was performed as described previously (8). In brief, the interstitial volume index $\left(I_{\text {Int }}\right)$ was determined by superposing a grid containing $100(10 \times 10)$ sampling points on photographs of 12 nonoverlapping cortical fields of silver-stained tissue $(\times 400)$ of each kidney. The number of points overlying interstitial space were counted. The indices of interstitial collagen deposition $\left(I_{\mathrm{col}}\right)$, tubular cell damage $\left(I_{\mathrm{TCD}}\right)$, and tubular dilatation $\left(I_{\text {Tdil }}\right)$ were assessed accordingly $(8)$.

Immunohistochemistry. All immunohistological studies were performed on paraffin-embedded sections as described (8). The following rat and rabbit Ab's were used as primary Ab's: rat anti-mCD45 (leukocytes, 1:100; PharMingen, San Diego, California, USA), rat anti-F4/80 (macrophages, 1:50; Serotec Ltd., Oxford, United Kingdom), rat anti-CD3 (lymphocytes, 1:50, Serotec Ltd.), rat anti-FSP1 (activated fibroblasts, 1:500; from F. Strutz) (16).

Isolation of renal cells for FACS analysis. A preparation of isolated renal cells including infiltrating leukocytes was obtained from obstructed and contralateral kidneys, as described previously (8). The resulting supernatant and blood samples taken from anesthetized mice by retrobulbar puncture were then labeled for flow cytometry. Samples were incubated with $5 \mu \mathrm{g} / \mathrm{ml}$ of mAb's against murine CCR2, murine CCR5, or the isotype control rat IgG2b (PharMingen), as described (17). To identify leukocyte subsets samples were incubated with the following 


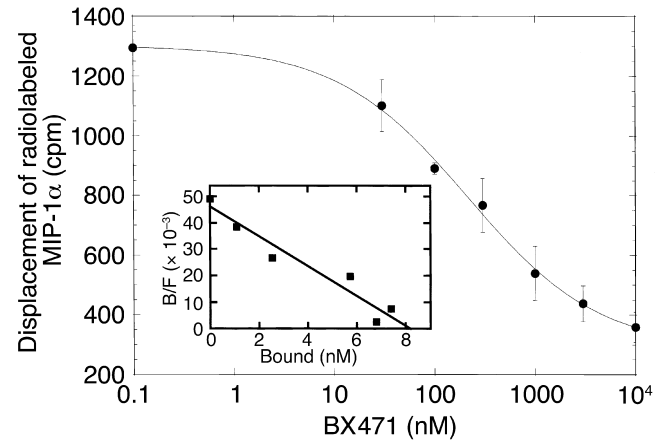

directly conjugated cell-specific Ab's: CD11b fluoresceinisothiocyanate (clone M1/70), CD4 allophycocyanin, and CD8 CyChrome (all PharMingen). Approximately 100,000 gated events were collected in each analysis.

Real-time quantitative RT-PCR. From each animal aliquots of both kidneys were snap-frozen in liquid nitrogen and stored at $-80^{\circ} \mathrm{C}$. RNA preparation and real-time RT-PCR on a TaqMan ABI 7700 Sequence Detection System (PE Biosystems, Weiterstadt, Germany) was performed as described (18). Controls consisting of $\mathrm{ddH}_{2} \mathrm{O}$ were negative for target and the housekeeper, GAPDH. The following oligonucleotide primers $(300 \mathrm{nM})$ and probes $(100 \mathrm{nM})$ were used: murine col-

\section{Figure 1}

Binding of BX471 to murine CCR1. The CCR1 antagonist, BX471, displaces radiolabeled MIP- $1 \alpha$ from murine CCR1. HEK cells transfected with murine CCR1 were incubated with ${ }^{125} \mathrm{I}-\mathrm{MIP}-1 \alpha / C C L 3$ in the presence of increasing concentrations of $\mathrm{BX} 471$. The binding reactions were terminated by centrifugation of cells as described previously (14). Binding shown represents specific binding from a typical experiment $(n=3)$. Nonspecific binding was $10 \%$ of total ${ }^{125}$ I-MIP- $1 \alpha / C C L 3$ added. Inset shows the Scatchard plot of the displacement data.

lagen I $\alpha 1$ (gb X 54876; bp 1984-2102): sense, 5'TGCTTTCTGCCCGGAAGA-3', antisense, $5^{\prime}$-GGGATGCCATCTCGTCCA- $3^{\prime}$, internal fluorescence-labeled probe (FAM), 5'-CCAGGGTCTCCCTTGGGTCCTACATCT-3'; murine GAPDH (gb M32599; bp 730-836): sense, 5'CATGGCCTTCCGTGTTCCTA-3', antisense $5^{\prime}$-ATGCCTGCTTCACCACCTTCT- $3^{\prime}$, internal fluorescence-labeled probe (VIC), 5'-CCCAATGTGTCCGTCGTGGATCTGA-3'. All probes were obtained from PE Biosystems.

Western blot. Kidneys were homogenized in RIPA buffer (Roche Molecular Biochemicals, Mannheim, Germany). Extracted proteins were boiled in loading buffer for 10 minutes, resolved by $8 \%$ SDS-PAGE, and trans-
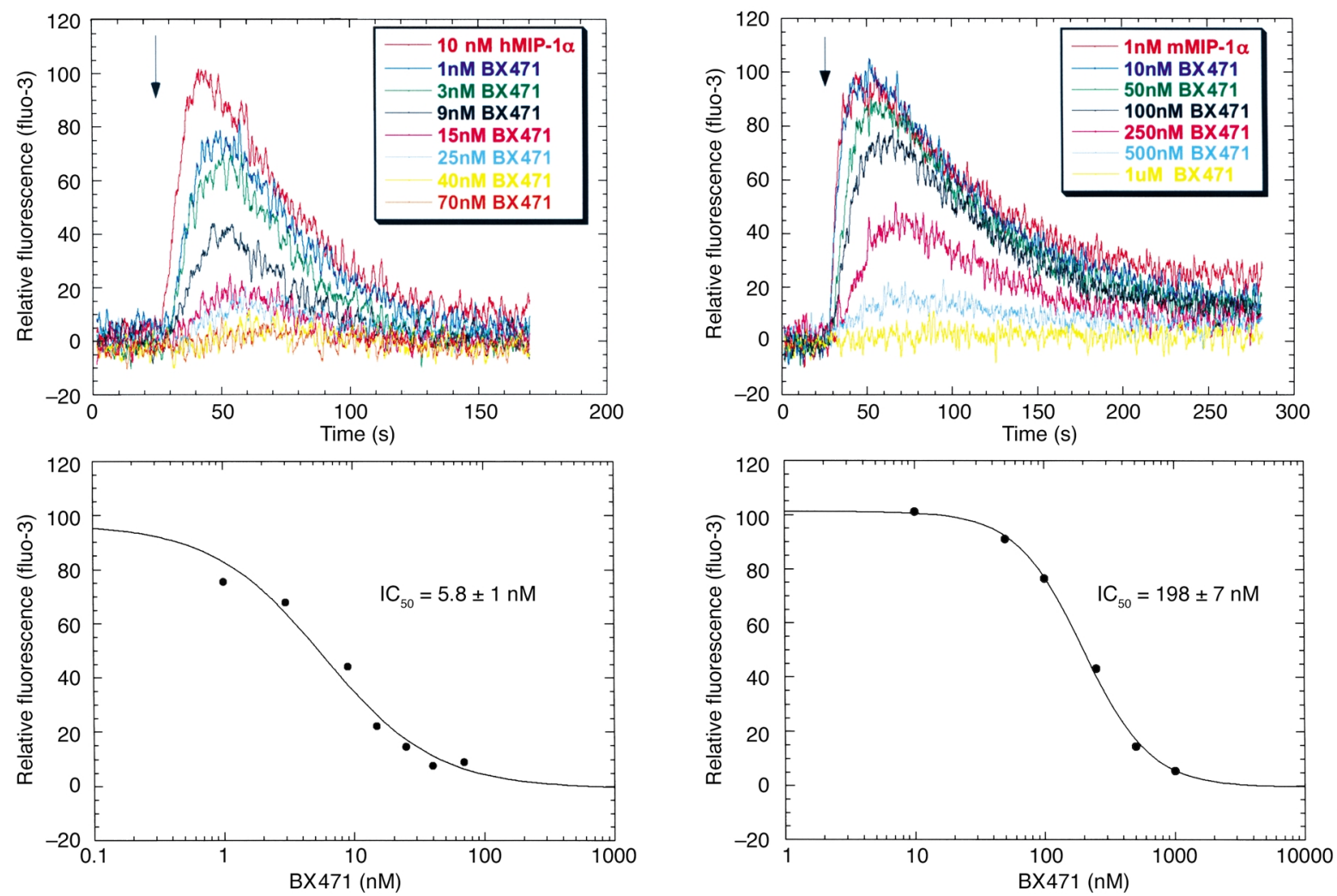

Figure 2

Cytosolic $\mathrm{Ca}^{2+}$ measurements in HEK 293 cells. BX471 inhibited the ability of MIP-1 $\alpha / C C L 3$ to increase Ca ${ }^{2+}$ transients in HEK 293 cells expressing human and murine CCR1. Fluo-3-loaded cells were pretreated with increasing concentrations of BX471 for 15 minutes and then stimulated with the CCR1 agonist, MIP-1 $\alpha / C C L 3$. The changes in fluorescence representing the changes in $\mathrm{Ca}^{2+}$ concentration were measured as indicated under Methods. 


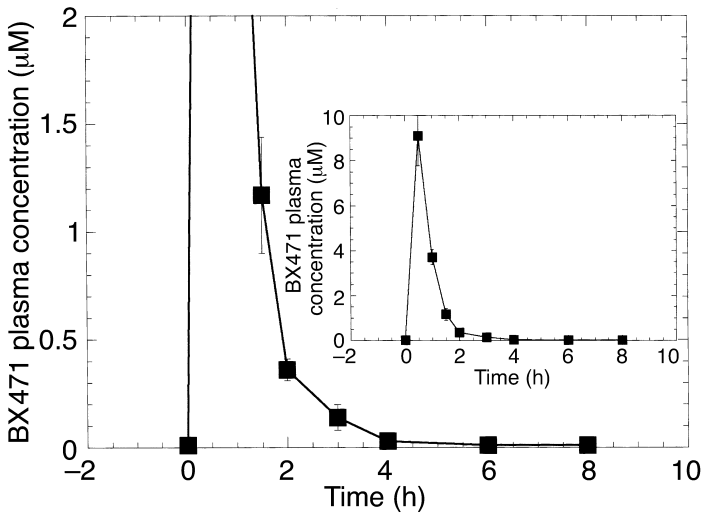

Figure 3

Pharmacokinetics of BX471 in mice. Plasma concentrations of BX471 following subcutaneous dosing in mice. Male mice $(n=4)$ received a single subcutaneus dose $(20 \mathrm{mg} / \mathrm{kg})$ of BX471 in $40 \%$ cyclodextrin. Blood plasma levels of BX471 were measured as described previously (9). Data are the mean \pm SEM.

ferred to an Immobilon-P membrane (Millipore, Eschborn, Germany). After blocking, the filter was incubated with the rabbit polyclonal anti-collagen I Ab (1:1,000; Chemicon International, Temecula, California, USA), and immune complexes were visualized using a peroxidase-conjugated $\mathrm{Ab}$ (1:5,000 in blocking solution; Jackson ImmunoResearch Laboratories, West Grove, Pennsylvania, USA) and then processed for detection by enhanced chemiluminescence (ECL; Amersham Pharmacia Biotech Europe, Freiburg, Germany).

Statistical analysis. Data were expressed as mean plus or minus SD. Comparison of groups was performed using univariate ANOVA, and post hoc Bonferroni's correction was used for multiple comparisons. Paired Student $t$ test was used for the comparison of single groups (RT-PCR and FACS data). A $P$ value of less than 0.05 was considered to indicate statistical significance.

\section{Results}

Binding of BX471 to murine CCR1. At first we have determined the affinity of BX 471 for mouse CCR 1 in competition binding studies. In competition binding experiments with HEK 293 cells expressing human CCR1, BX471 was able to displace ${ }^{125}$ I-MIP-1 $\alpha /$ CCL3 binding in a concentration-dependent manner with a $K_{\mathrm{I}}$ of $1.0 \pm 0.03 \mathrm{nM}$, which is similar to the $K_{\mathrm{I}}$ for MIP- $1 \alpha / C C L 3$ of $2 \mathrm{nM}$ (14). Here we show that BX471 was also able to displace ${ }^{125}$ I-MIP- $1 \alpha /$ CCL3 binding to mouse CCR1 in a concentration-dependent manner with a $K_{\mathrm{I}}$ of $215 \pm 46 \mathrm{nM}$ (Figure 1). Because this was about 20-fold lower compared with the binding to human CCR1, we studied the specificity of BX471 to murine CCR1 compared with other murine chemokine receptors that are known to be involved in the UUO model (8). BX471 did not crossreact with CCR2, CCR5, and CXCR4 at concentrations greater than $50 \mu \mathrm{M}$, giving at least a 250 -fold specificity for CCR1 (data not shown).
Cytosolic $\mathrm{Ca}^{2+}$ measurements in HEK 293 cells. To demonstrate that BX471 is a functional antagonist for mouse CCR1, we measured the ability of the compound to inhibit agonist-induced $\mathrm{Ca}^{2+}$ mobilization in CCR1expressing cells. As shown in Figure 2, MIP- $\alpha /$ CCL3 induced a rapid and transient increase in intracellular $\mathrm{Ca}^{2+}$ in both human and mouse CCR1. Increasing concentrations of $\mathrm{BX} 471$ inhibited the $\mathrm{Ca}^{2+}$ transients induced by MIP-1 $\alpha /$ CCL 3 in both human and mouse CCR1 with $\mathrm{IC}_{50}$ of $5.8 \pm 1 \mathrm{nM}$ and $198 \pm 7 \mathrm{nM}$, respectively, demonstrating functional antagonism for CCR1 (Figure 2). When given alone, the compound did not induce $\mathrm{Ca}^{2+}$ transients, indicating that the compound has no intrinsic agonistic activity (data not shown).

Determination of pharmacokinetic parameters in mice. The pharmacokinetic profile of BX471 was examined in conscious mice. BX471 was administered to mice at 20 $\mathrm{mg} / \mathrm{kg}$ in a vehicle of $40 \%$ cyclodextrin/saline by subcutaneous injection. The plasma samples were prepared as described previously, and compound concentrations in
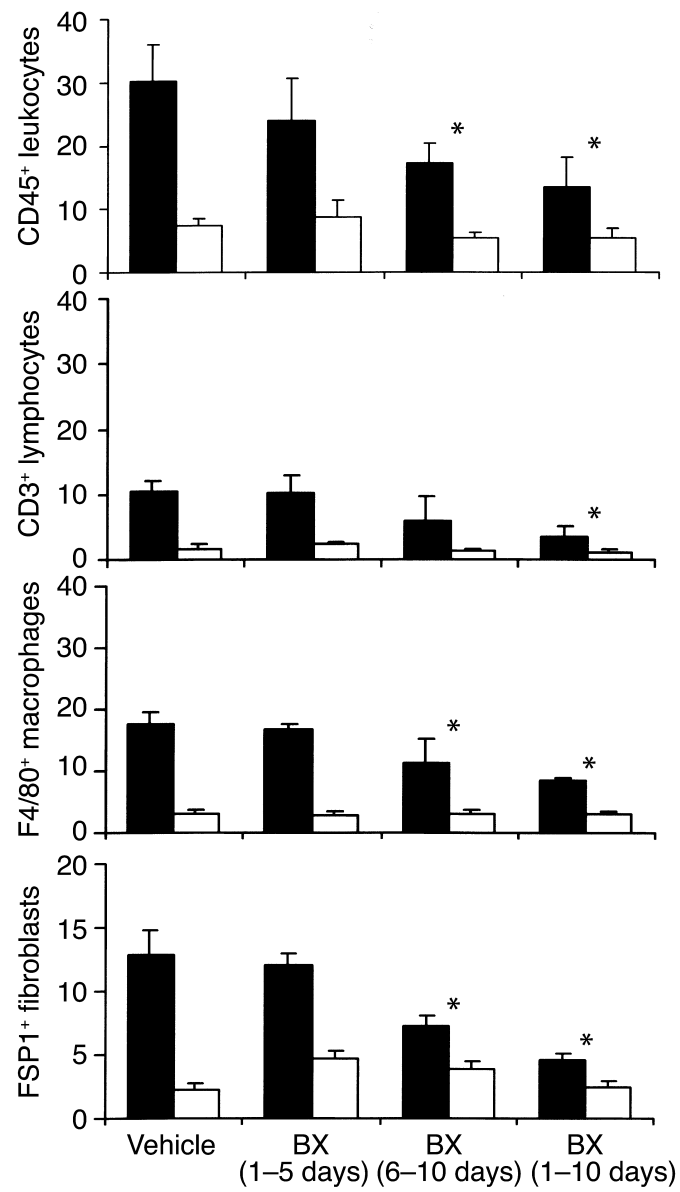

Figure 4

Accumulation of interstitial cells after UUO. Quantitative analysis of cell infiltration 10 days after UUO in obstructed (black bars) and contralateral unobstructed kidneys (white bars). Values are means \pm SD of cell counts per hpf. Note that treatment with BX471 for 10 days as well as from days 6-10 reduced infiltration of $\mathrm{CD} 45^{+}$leukocytes, $\mathrm{CD} 3^{+}$lymphocytes, $\mathrm{F} 4 / 80^{+}$macrophages, and $\mathrm{FSP} 1^{+}$fibroblasts compared with vehicle controls. BX471 given from day $0-5$ had no effect. ${ }^{*} P<0.05$. 


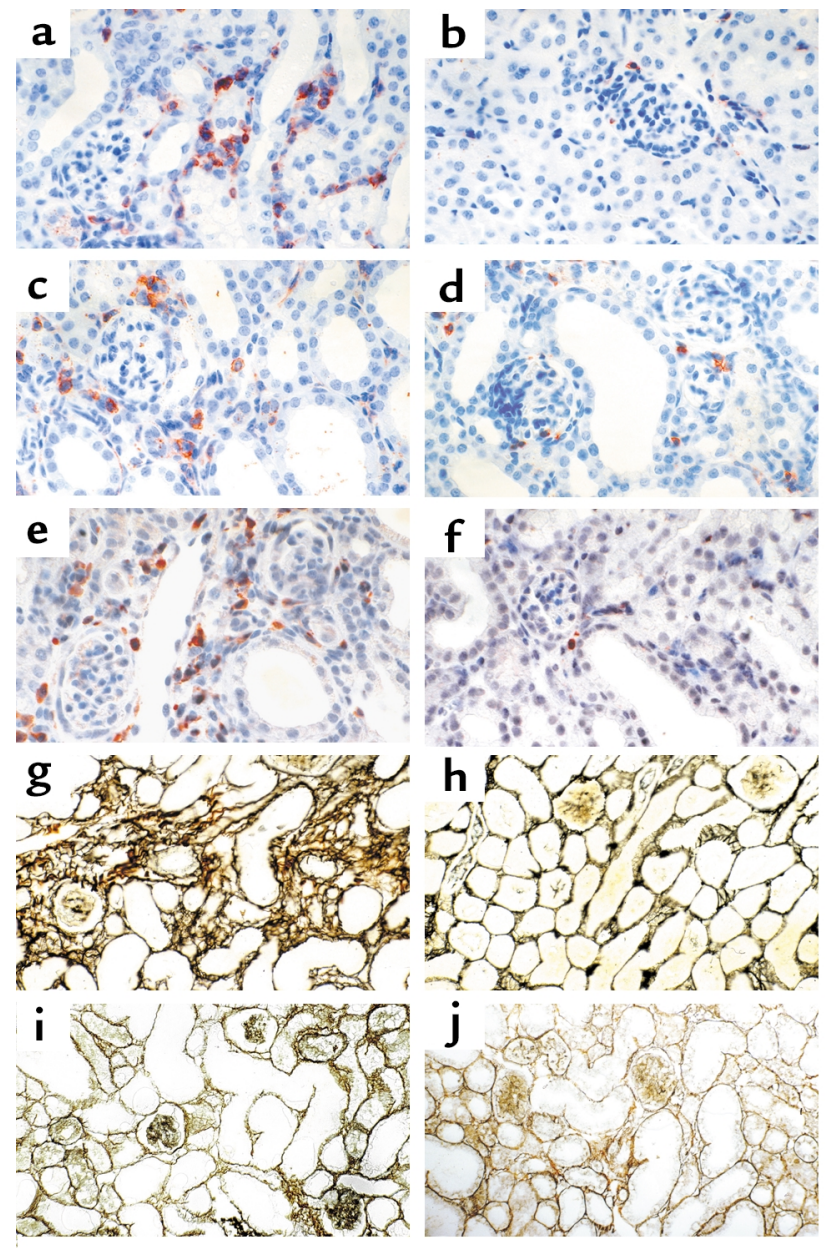

the plasma were determined by HPLC (14). As shown in Figure 3, BX471 reached peak plasma levels of $9 \mu \mathrm{M}$ by around 30 minutes, and this rapidly declined to approximately $0.4 \mu \mathrm{M}$ after 2 hours. From 4 to 8 hours the drug plasma levels dropped to $0.1 \mu \mathrm{M}$ or lower. Based on these data we decided to dose the animals subcutaneously three times a day with $20 \mathrm{mg} / \mathrm{kg}$ of BX471.

\section{Parameters of tubulointerstitial disease after UUO}

Infiltrating leukocytes. At day 10 after UUO, a prominent cell infiltrate of CD45-positive leukocytes, CD3-positive lymphocytes, and F4/80-positive macrophages was localized to the peritubular interstitium in vehicle-

\section{Figure 6}

Renal chemokine receptor expression after UUO. Real-time RT-PCR was performed using total renal RNA of obstructed (UUO) and contralateral (CLK) kidneys of four to six mice 10 days after ureter ligation. Levels of mRNA expression for CCR1, CCR2, and CCR5 are expressed in relation to renal GAPDH mRNA expression as indicated in Methods. The expression in UUO kidneys of control mice is set as 1. (a) A marked increase of mRNA for all three receptors was noted in UUO kidneys compared with CLK of vehicle controls. (b) Treatment with $B \times 471$ for 10 days resulted in a significant decrease of CCR 1 and CCR5 mRNA expression $(P=0.005$ and $P=0.03$, respectively) compared with UUO kidneys of vehicle controls. For CCR2 the difference was not significant $(P=0.07) .{ }^{*} P<0.05$.

\section{Figure 5}

Leukocyte infiltration and renal fibrosis after UUO histology. Cortical renal sections were stained for $\mathrm{CD} 45^{+}$leukocytes. At 10 days after UUO (a) leukocytes accumulated in the peritubular interstitium in obstructed kidneys compared with unobstructed control kidneys (b). Treatment with BX471 from day 6 to 10 (c), as well as treatment for 10 days $(\mathbf{d})$, reduced infiltration of CD45-positive leukocytes in UUO kidneys compared with $\mathbf{a}$ and $\mathbf{b}$. No reduction was noted in UUO kidneys of mice treated with BX471 from days 0 to 5 (not shown). The accumulation of FSP1-positive fibroblasts in the peritubular interstitium 10 days after $\mathrm{UUO}(\mathbf{e})$ was reduced when BX471 was given for 10 days (f) and from day 6 to 10 (latter not shown). Cortical renal sections were also silver stained to assess interstitial volume and fibrous tissue deposition. Note a marked widening of the interstitial space with deposition of fibrous tissue in areas of dilated tubules in obstructed kidneys at 10 days (g) compared with unobstructed control kidneys (h) of vehicle controls. Treatment with BX471 from days 6 to 10 reduced interstitial volume and matrix deposition (i), as well as BX471 treatment for 10 days (j), compared with $\mathbf{g}$. No reduction was noted in UUO kidneys of mice treated with BX471 at days 0-5 (not shown). Tubular dilatation and tubular epithelial cell damage remained unchanged in all groups (original magnification $\times 400$ ).

treated controls (Figures 4 and 5). To further characterize the chemokine receptor expression of the cellular infiltrate we performed real-time RT-PCR for CCR1, CCR2, and CCR5 using renal RNA. There was a marked increase of mRNA of all three chemokine receptors in obstructed compared with unobstructed kidneys (Figure 6). To characterize the receptor expression on infiltrating $\mathrm{T}$ cells, FACS analysis was performed on renal cell isolates; $64 \%$ of CD8 T cells infiltrating the obstructed kidney were positive for CCR5 compared with $42 \%$ in the contralateral kidney (not shown) and $17 \%$ in the peripheral blood (Figure 7). Kidneys of healthy C57BL/ 6 mice not injected with the vehicle contained 21\% CCR5-positive CD8 T cells (not

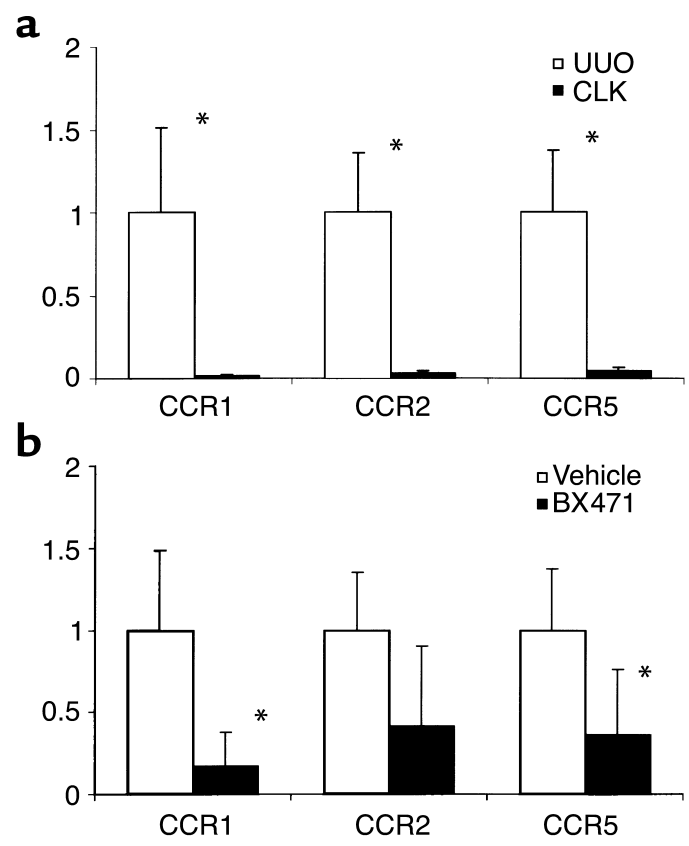



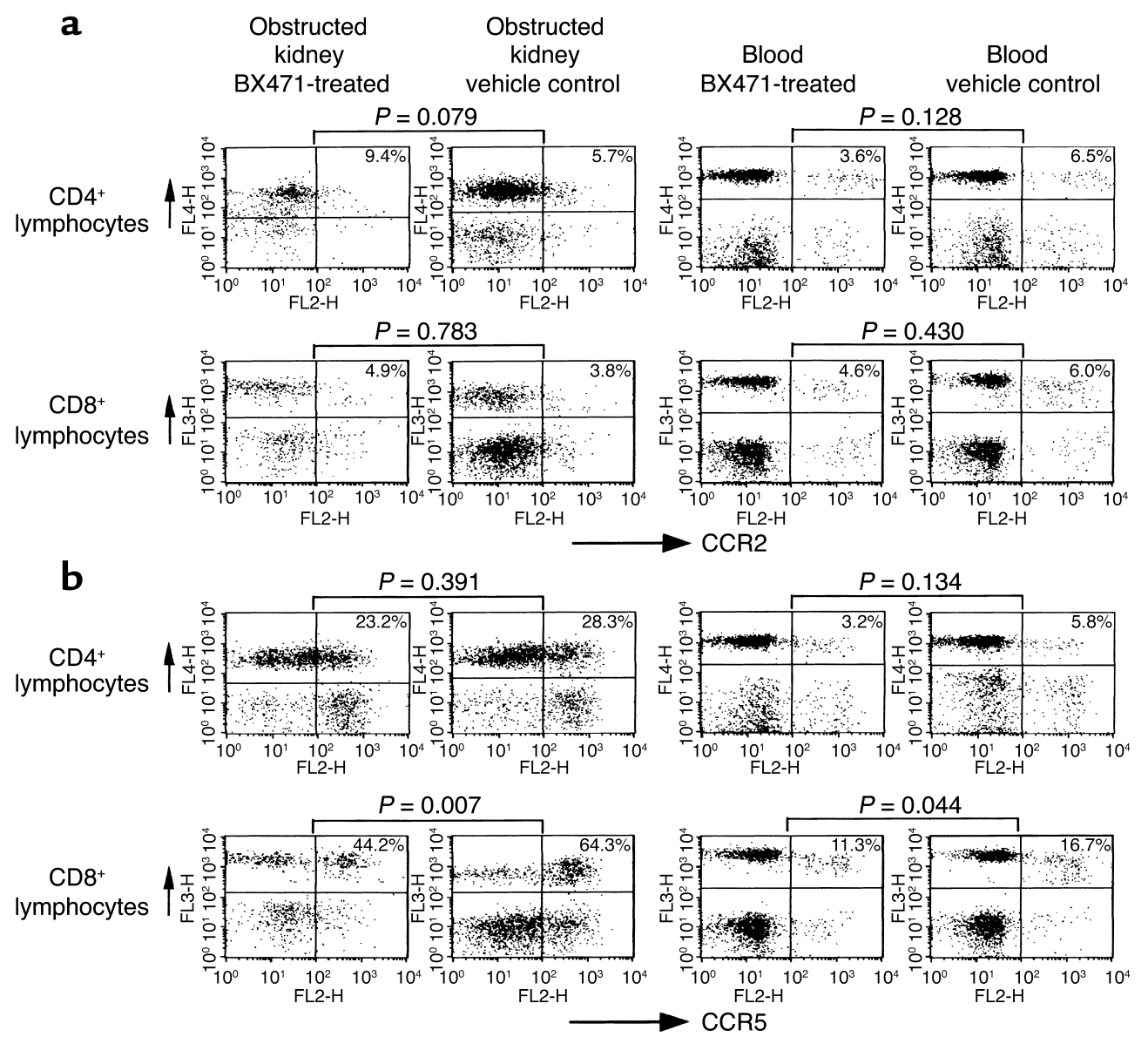

\section{Figure 7}

CCR2 and CCR5 expression on infiltrating leukocytes. Flow cytometric analysis of CCR2 (a) and CCR5 (b) expression on T cells isolated from obstructed kidneys and blood of BX471-treated mice and vehicle controls. Treatment with BX471 results in a significant decrease of CCR5-positive CD8 ${ }^{+}$lymphocytes infiltrating the obstructed kidneys and, to a lower extent, also in the peripheral blood. No significant changes were seen for CCR5-positive CD4 cells or CCR2-positive cells in kidneys or blood from BX471- or vehicle-treated mice. Dot blots are representative for four to six separate sets of experiments. Numbers given are means of five to nine mice analyzed in each group.

shown). There was no increase of CCR5-positive CD4 cells or CCR2-positive CD4 or CD8 cells in UUO kidneys compared with contralateral kidneys or peripheral blood in mice of vehicle-treated controls.

Fibrosis. As a cellular marker of fibrosis, the amount of FSP1-positive fibroblasts was assessed by immunohistochemistry. Ten days after UUO fibroblasts accumulated in the peritubular interstitium in areas of marked tubular dilatation (Figures 4 and 5). Morphometric analysis of silver-stained renal sections revealed a marked increase of interstitial volume and collagen deposition in obstructed kidneys compared with unobstructed control kidneys (Figure 8). Using quantitative real-time TaqMan RT-PCR, mRNA for collagen I $\alpha 1$ was also found to be upregulated after UUO compared with contralateral control kidneys (Figure 9). To confirm the increase of collagen expression we performed Western blot analysis with a specific $\mathrm{Ab}$ for collagen I $\alpha 1$. Ten days after surgery obstructed kidneys of collagen I, protein expression was markedly increased compared with contralateral controls (Figure 9).
BX471 reduces leukocyte infiltration

and renal fibrosis after UUO

Infiltrating leukocytes. Mice treated with $20 \mathrm{mg} / \mathrm{kg}$ of the CCR1 antagonist BX471 for 10 days showed a reduction of interstitial CD45 positive leukocytes of approximately $55 \%$ as compared with vehicle-treated mice (Figures 4 and 5). The tubulointerstitial infiltration of CD3-positive lymphocytes and F4/80-positive macrophages was reduced by $64 \%$ and $50 \%$, respectively (Figure 4). Using FACS analysis of isolated infiltrating cells this decrease was accompanied by a significant reduction of CCR5-positive CD8 T cells in UUO kidneys of BX471-treated mice as compared with kidneys of vehicle controls ( $44.2 \%$ vs. $64.3 \%, P=0.007$; Figure 7 ). BX471 had a borderline significant effect on the number of CCR5-positive CD8 cells in the peripheral blood $(11.3 \%$ vs. $16.7 \%, P=0.044)$. No significant effect was noted on the number of CCR5-positive CD4 cells as well as on CCR2-positive CD4 or CD8 T cells in peripheral blood and obstructed or unobstructed kidneys. Quantitative RT-PCR revealed a significant reduction of renal CCR1 and, to a lesser extent, CCR5 mRNA 

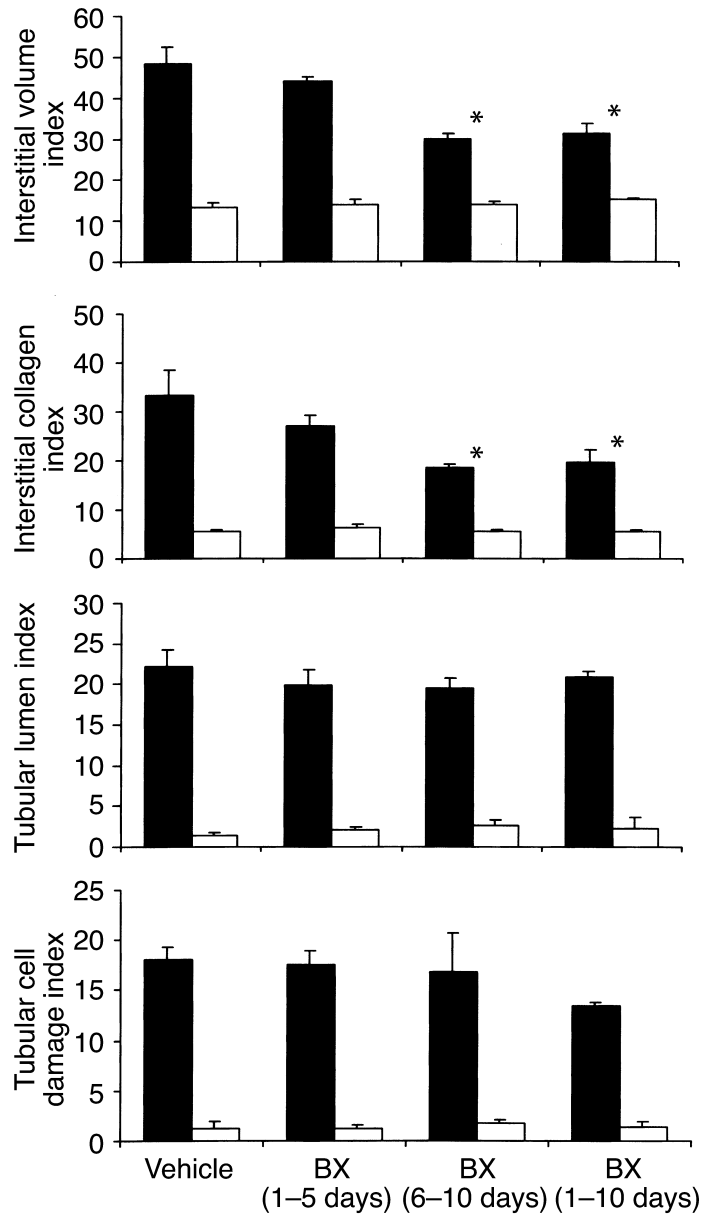

expression (Figure 6). There was a trend to a decrease of renal CCR 2 mRNA expression, although this did not reach statistical significance.

Fibrosis. BX471 reduced the amount of FSP1-positive cells by $65 \%$ in UUO kidneys as compared with vehicle controls (Figures 4 and 5). Morphometrically, interstitial volume and collagen were also reduced by $25 \%$ and $33 \%$, respectively (Figure 8 ). Collagen I $\alpha 1$ mRNA expression as assessed by Taqman RT-PCR was significantly reduced in obstructed kidneys of BX471-treated mice compared with those of vehicle controls (Figure 9, $P<0.05$ ). Western blot analysis using a specific collagen I Ab confirmed the

\section{Figure 8}

Histopathological damage after UUO: morphometry data. Morphometric analysis of cortical renal sections 10 days after UUO in obstructed kidneys (black bars) and contralateral unobstructed kidneys (white bars). Values are means \pm SD of cell counts per hpf. Note that treatment with BX471 for 10 days as well as from days 6 to 10 significantly reduced the interstitial volume index and collagen index compared with vehicle controls. BX471 given from day 0 to 5 had no effect on interstitial volume and collagen index. The indices of tubular lumen and tubular cell damage were not affected by either treatment regimen.

decrease of collagen I protein in renal extracts of BX471-treated mice compared with vehicle-treated controls (Figure 9).

Tubular damage. In contrast to the changes in the interstitium, parameters of tubular dilatation and epithelial cell damage were identical between BX471treated mice and vehicle controls (Figures 5 and 8). No changes in interstitial cell counts or morphometric parameters were observed in contralateral kidneys.

\section{Delayed BX471 treatment reduces renal} fibrosis after UUO to the same extent

When BX471 was administered starting at day 6 after UUO, a time point where a substantial number of interstitial leukocytes was already present $(16.2 \pm 3.1$ cell per hpf), the beneficial effects on leukocyte infiltration and renal fibrosis were still observed.

Infiltrating lenkocytes. In mice treated with BX 471 from day 6 a reduction of interstitial CD45-positive leukocytes of about $45 \%$ was observed at day 10 compared with vehicle controls of the same time point (Figure 4 and 5). Both macrophages and lymphocytes contributed to this reduction, although only the F4/80-positive cell count reached statistical significance compared with UUO kidneys of vehicle-controls. Compared with vehicle controls sacrificed on day 6 , mice treated from day 6 to 10 revealed no further increase of interstitial leukocyte counts (controls, day 6, $16.2 \pm 3.1$, vs. BX471, day $6-10,17.3 \pm 5.4$ cell per hpf).

Fibrosis. The reduced leukocyte infiltration in BX471treated mice correlated with a decrease of interstitial fibrosis. Renal interstitial fibroblasts were reduced by $45 \%$ compared with vehicle-treated controls of day 10 (Figures 4 and 5). Morphometrically, interstitial

\section{Figure 9}

Collagen I mRNA and protein expression. (a) Renal collagen mRNA expression was determined by real-time RT-PCR. Collagen I $\alpha 1$ mRNA was analyzed as the ratio to GAPDH template and compared with the unligated kidney. Collagen I expression of the vehicle group is set as 1 . A significant decrease of collagen I $\alpha 1$ mRNA was found in BX471-treated animals (treatment from day 0 to 10) compared with vehicle-treated mice. (b) Levels of collagen I were determined by Western blot analysis. Protein extractions of two obstructed (UUO) and two unobstructed contralateral kidneys (CLK) of BX471-treated mice (lanes 1 and 2) were compared with vehicle controls (lanes 3 and 4). Note an increase of collagen I in UUO compared with CLK kidneys. Treatment with BX471 for 10 days reduced collagen I protein expression in UUO kidneys compared with control UUO kidneys.

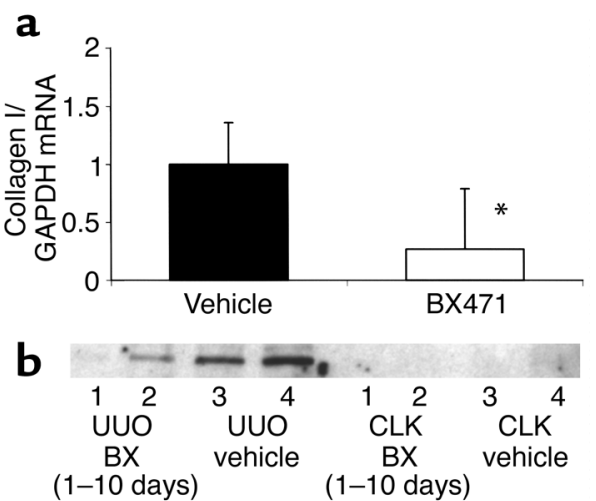


volume and collagen were also decreased in these groups by $25 \%$ or $33 \%$, respectively (Figures 5 and 8 ).

Tubular damage. Tubular dilatation and tubular cell damage were not affected by delayed BX471 treatment (Figures 5 and 8). No changes in interstitial cell counts or morphometric parameters were observed in contralateral kidneys.

Early BX471 treatment does not affect renal fibrosis. BX471 given from day 1 to day 5 after UUO had no significant effect on leukocyte infiltration and fibroblast accumulation compared with mice treated with the vehicle only. The indices of tubular dilatation and tubular cell damage were similar in all groups (Figures 4, 5, and 8).

\section{Discussion}

The UUO model allows the study of renal fibrosis independent of an ongoing systemic inflammatory disease. After UUO, the expression of CCR1, CCR2, and CCR5 mRNA correlates with interstitial leukocyte infiltration, fibroblast proliferation, and collagen expression (8). We therefore used the UUO model to study the effect of antagonizing CCR1 by BX471, a recently developed small molecule antagonist against human CCR1. We found that BX471 has the same antagonistic properties on murine CCR1 as reported for other species $(9,10,11$, 13); $20 \mathrm{mg} / \mathrm{kg} \mathrm{BX} 471$ three times a day were required to effectively antagonize the mouse CCR 1 receptor, which we show has a $K_{\mathrm{I}}$ of $215 \mathrm{nM}$ compared with $1 \mathrm{nM}$ for human CCR1. BX471 substantially reduced the amount of interstitial leukocyte and fibroblast accumulation, as well as interstitial fibrosis and collagen I expression. The reduction of infiltrating leukocytes correlated with a reduction of renal CCR1 and to a lesser extent of CCR5 mRNA expression. Most interestingly, delayed BX471 treatment starting at 6 days after UUO was as effective as treatment started at the initiation of obstruction.

BX471 reduces interstitial leukocyte infiltration and fibrosis after UUO. The reduction of leukocyte infiltration and chemokine receptor expression with BX471 is most likely a consequence of blocking CCR1-dependent leukocyte adhesion and transmigration. CCR1 is expressed on circulating macrophages and lymphocytes in the peripheral blood of mice and humans (19). Chemokine ligand binding to CCR1 results in an upregulation of $\beta 2$ integrin, which is required for firm leukocyte adhesion to activated endothelium during the rolling phase (9). In addition, CCR1 mediates transmigration through the endothelium-basement membrane barrier into the interstitial space (20). Both mechanisms have been shown to be inhibited by BX471 in a dose-dependent manner under conditions of shear flow $(9,20)$. As we show here, BX471 inhibits MIP-1 $\alpha / C C L 3$ binding to murine CCR1 and blocks receptor activation as determined by $\mathrm{Ca}^{2+}$ mobilization. The mechanism is thought to be a competitive antagonist of ligand binding instead of ligand inactivation since BX471 does not affect MIP-1 $\alpha / C C L 3$ binding to CCR5 (9).

Other in vivo studies have also demonstrated a beneficial effect of BX471 on the outcome of disease models that involve the infiltration of CCR1-positive mononuclear cells. In rats, BX471 dose dependently improved the severity of the experimental allergic encephalomyelitis (EAE) model (13). In combination with cyclosporine a similar dose of BX471 effectively prolonged rat heart transplant survival, which correlated with a reduction of mononuclear cell infiltrates (10). In a rabbit model of renal transplantation BX471 was similar to cyclosporine in its ability to prevent transplant rejection and to improve mortality (11). Taken together, these data illustrate that CCR1 is involved in the infiltration of mononuclear cells during the progression of chronic inflammatory disorders. Tokuda et al. used a neutralizing $\mathrm{Ab}$ against murine CCR1 in the bleomycin-induced pulmonary fibrosis model (21). Three doses of the antiCCR1 Ab, but not of an anti-CCR2 Ab, reduced pulmonary mononuclear cell infiltration and fibrosis 10 days after induction of disease. We therefore conclude that blocking CCR1 either by specific Ab's or a nonpeptide antagonist can reduce organ fibrosis by reducing leukocyte infiltration.

Due to a lack of appropriate Ab's against murine CCR1 that work in FACS or immunohistochemistry, we could not directly study the amount of CCR1-positive cells infiltrating the kidney with or without BX471 treatment. However, using RT-PCR, a reduction of renal CCR1 and, to a lesser extent, CCR5 mRNA expression in the UUO kidneys of BX471-treated mice indicates that BX471 reduced the renal accumulation of CCR1positive cells. The mRNA expression data were confirmed for CCR5-positive T cells by FACS analysis, which demonstrated a reduction of CCR5-positive CD8 cells after UUO by BX471 in the kidney and to a lesser extent in the peripheral blood. The role of the latter needs further clarification, but the renal data suggest that the infiltration of CCR5-positive cells is modulated by CCR1 in vivo. CCR 1 and CCR 5 are coexpressed in the minority of circulating $T$ cells, but $T$ cells can upregulate CCR5 during activation (22). Circulating monocytes express CCR1, but only a small subpopulation coexpresses CCR5 (23). CCR1-positive monocytes upregulate CCR5 during activation and maturation toward the tissue macrophage phenotype (23). Furthermore, in vitro studies under shear flow conditions have shown that adhesion and transendothelial chemotaxis of CCR5-positive mononuclear cells can be blocked with BX471 (20). Our finding that BX471 also reduces the renal infiltration of CCR5-positive CD8 cells after UUO indicates that CCR1 modulates infiltration of CCR5-positive cells in vivo also. In various experimental models CCR5-positive T cells were found to be a marker for lymphocytes that are involved in chronic inflammatory lesions $(5,16,24,25)$. In human renal biopsy studies CCR5-positive $\mathrm{T}$ cells predominantly locate in the tubulointerstitium and correlate with disease activity (25). In renal transplant rejection, there is a marked increase of CCR5-positive leukocytes in the tubulointerstitium (5). A role for CCR5-positive leukocytes in renal transplant rejection is also supported by 
the finding that transplant recipients who carry the CCR5- $\Delta 32 / \Delta 32$ mutation have a longer transplant survival compared with the control population (26).

The decrease in renal fibrosis also involved a reduction of interstitial fibroblast accumulation. After UUO, interstitial fibroblasts receive stimuli for proliferation and matrix production from at least three sources: activated tubular epithelial cells, infiltrating leukocytes, and interstitial cell hypoxia following the distension of the interstitial space (27). The observed effects of BX471 are particularly interesting in the UUO model in view of the unchanged tubular epithelial cell damage during progressive tubular dilatation. The expression of CCR 1 by fibroblasts has not been reported, but a direct effect of BX471 on these cells cannot be excluded. However, it is most likely that the reduction of mononuclear cell infiltrates induced less fibroblast proliferation by a reduction of secreted proinflammatory cytokines known to stimulate fibroblast proliferation.

Delayed onset of BX471 treatment also reduces leukocyte infiltration and fibrosis after UUO. In most experimental studies antagonists are given from the start of the lesion. In an attempt to more closely mimic the clinical situation we tested the initiation of treatment at a time point where renal fibrosis was already visible (6). BX471 given from day 6 reduced the progressive increase of leukocyte infiltration and renal fibrosis to the same extent as when treatment was initiated immediately after UUO. This observation is surprising because BX471 seemed to halt the amount of interstitial leukocytes and fibrosis when BX471 was given from day 6 . However, besides its effects on leukocyte infiltration, BX471 could have additional roles in leukocyte homeostasis such as regulating leukocyte apoptosis within the kidney or emigration of leukocytes out of the kidney into blood or lymph vessels, an issue that needs further clarification. At present, to our knowledge this is the first study that demonstrates a beneficial effect of treatment with a chemokine receptor antagonist on the progression of established fibrosis in vivo. In contrast BX471 treatment limited to the first 5 days after UUO was unable to reduce fibrosis at day 10 . In view of the pharmacokinetic profile of BX471 these data argue for a continued administration of BX471 in vivo.

In summary, CCR1 appears to play an important role in mediating the infiltration of mononuclear cells and subsequent renal fibrosis after UUO. BX471 reduced the infiltration of macrophages, lymphocytes, and, more specifically, CCR5-positive CD8 T cells infiltrating the obstructed kidney. Most likely this is achieved by blocking CCR1-dependent cell adhesion and transmigration. Since delayed treatment with BX471 had the same effect, we propose that CCR1 blockade may offer a new therapeutic strategy to halt renal fibrosis in obstructive nephropathy and perhaps in other chronic nephropathies leading to end-stage renal disease.

\section{Acknowledgments}

The work was supported by grants from the WilhelmSander Foundation to H.J. Anders, the University of Munich to V. Vielhauer, the Deutsche Forschungsgesellschaft to M. Mack, and the German Human Genome Project, the Else-Kroener-Fresenius Foundation, and the EU Concerted Action to M. Kretzler.

1. Becker, G.J., and Hewitson, T.D. 2000. The role of tubulointerstitial injury in chronic renal failure. Curr. Opin. Nephrol. Hypertens. 9:133-138.

2. Zeisberg, M., Strutz, F., and Muller, G.A. 2001. Renal fibrosis: an update. Curr. Opin. Nephrol. Hypertens. 10:315-320.

3. Zlotnik, A., and Yoshie, O. 2000. Chemokines: a new classification system and their role in immunity. Immunity. 12:121-127.

4. Segerer, S., Nelson, P.J., and Schlöndorff, D. 2000. Chemokines, chemokine receptors, and renal disease: from basic science to pathophysiologic and therapeutic studies. J. Am. Soc. Nephrol. 11:152-176.

5. Segerer, S., et al. 2001. Expression of chemokines and chemokine receptors during human renal transplant rejection. Am. J. Kidney Dis. 37:518-531.

6. Furuichi, K., et al. 2000. Distinct expression of CCR1 and CCR5 in glomerular and interstitial lesions of human glomerular diseases. Am.J. Nephrol. 20:291-299.

7. Diamond, J.R., Ricardo, S.D., and Klahr, S. 1998. Mechanisms of interstitial fibrosis in obstructive nephropathy. Semin. Nephrol. 18:594-602.

8. Vielhauer, V., et al. 2001. Obstructive nephropathy in the mouse: progressive fibrosis correlates with tubulointerstitial chemokine expression and accumulation of CC-chemokine receptor- 2 and -5 positive leukocytes. J. Am. Soc. Nephrol. 12:1173-1187.

9. Liang, M., et al. 2000. Identification and characterization of a potent, selective, and orally active antagonist of the CC chemokine receptor-1. J. Biol. Chem. 275:19000-19008.

10. Horuk, R., et al. 2001. A nonpeptide functional antagonist of the CCR1 chemokine receptor is effective in rat heart transplant rejection. J. Biol. Chem. 276:4199-4204.

11. Horuk, R., et al. 2001. CCR1-specific non-peptide antagonist: efficacy in a rabbit allograft rejection model. Immunol. Lett. 76:193-201.

12. Conklin, B.R., Farfel, Z., Lustig, K.D., Julius, D., and Bourne, H.R. 1993. Substitution of three amino acids switches receptor specificity of Gq alpha to that of Gi alpha. Nature. 363:274-276.

13. Coward, P., Chan, S.D., Wada, H.G., Humphries, G.M., and Conklin, B.R. 1999. Chimeric $G$ proteins allow a high-throughput signaling assay of Gi-coupled receptors. Anal. Biochem. 270:242-248.

14. Hesselgesser, J., et al. 1998. Identification and characterization of small molecule functional antagonists of the CCR1 chemokine receptor. J. Biol. Chem. 273:15687-15692.

15. Liang, M., et al. 2000. Species selectivity of a small molecule antagonist for the CCR1 chemokine receptor. Eur. J. Pharmacol. 389:41-49.

16. Strutz, F., et al. 1995. Identification and characterization of a fibroblast marker: FSP1. J. Cell Biol. 130:393-405.

17. Mack, M., et al. 2001. Expression and characterization of the chemokine receptors CCR2 and CCR5 in mice. J. Immunol. 166:4697-4704.

18. Anders, H.J., et al. 2001. Chemokine and chemokine receptor expression during initiation and resolution of immune complex glomerulonephritis. J. Am. Soc. Nephrol. 12:919-931.

19. Murphy, P.M., et al. 2000. International union of pharmacology. XXII. Nomenclature for chemokine receptors. Pharmacol. Rev. 52:145-176.

20. Weber, C., et al. 2001. Specialized roles of the chemokine receptors CCR1 and CCR5 in the recruitment of monocytes and $\mathrm{T}(\mathrm{H}) 1-\mathrm{like} / \mathrm{CD} 45 \mathrm{RO}(+)$ T cells. Blood. 97:1144-1146.

21. Tokuda, A., et al. 2000. Pivotal role of CCR1-positive leukocytes in bleomycin-induced lung fibrosis in mice. J. Immunol. 164:2745-2751.

22. Sallusto, F., et al. 1999. Switch in chemokine receptor expression upon TCR stimulation reveals novel homing potential for recently activated T cells. Eur. J. Immunol. 29:2037-2045.

23. Kaufmann, A., Salentin, R., Gemsa, D., and Sprenger, H. 2001. Increase of CCR1 and CCR5 expression and enhanced functional response to MIP-1 alpha during differentiation of human monocytes to macrophages. J. Leukoc. Biol. 69:248-252.

24. Qin, S., et al. 1998. The chemokine receptors CXCR3 and CCR5 mark subsets of $\mathrm{T}$ cells associated with certain inflammatory reactions. J. Clin. Invest. 101:746-754.

25. Segerer, S., Mack, M., Regele, H., Kerjaschki, D., and Schlöndorff, D. 1999. Expression of the C-C chemokine receptor 5 in human kidney diseases. Kidney Int. 56:52-64.

26. Fischereder, M., et al. 2001. CC chemokine receptor 5 and renal-transplant survival. Lancet. 357:1758-1761.

27. Zeisberg, M., Strutz, F., and Müller, G.A. 2000. Role of fibroblast activation in inducing interstitial fibrosis. J. Nephrol. 3:S111-S120. 